\title{
Acute toxicity test of copper pyrithione on Javanese medaka and the behavioural stress symptoms
}

\begin{abstract}
This study was conducted to investigate the median lethal concentration (LC50) of copper pyrithione $(\mathrm{CuPT})$ at $96-\mathrm{hr}$ exposure on adult Javanese medaka (Oryzias javanicus) in revealing toxicological effects of CuPT contamination in the tropical area. Wild stock fishes were acclimatized for 14-days prior analysis. Triplicate of test tanks for seven test concentrations were placed with ten fishes each, this includes two control tanks. The behaviour of the tested fishes was manually observed through a camera. The LC50 of CuPT at $96-\mathrm{h}$ was found to be $16.58 \mathrm{mg} / \mathrm{L}$. Tested fishes swam slowly in vertical movement and swam fast towards food during feeding time as the sign of stress behaviour. Meanwhile, fishes in the two control groups swam actively in a horizontal manner and no excitement during feeding time. No mortality in control groups. Results indicate CuPT to be toxic to Javanese medaka at low concentration and caused behavioural stress.
\end{abstract}

Keyword: Copper pyrithione; Antifouling; Biocide; Javanese medaka; Acute toxicity 